\title{
Poly(sulfur-random-(1,3-diisopropenylbenzene)) Based Mid-Wavelength Infrared Polarizer: Optical Property Experimental and Theoretical Analysis
}

Aaron J. Berndt, ${ }^{\mathrm{a}, \dagger}$ Jehwan Hwang, ${ }^{\mathrm{b}, \dagger}$ Md Didarul Islam, ${ }^{\mathrm{c}}$ Amy Sihn, ${ }^{\mathrm{d}, \mathrm{e}}$ Augustine M. Urbas, ${ }^{\mathrm{d}, *}$ Zahyun Ku,,${ }^{\mathrm{d}, *}$ Sang J. Lee, ${ }^{\mathrm{i}}$ David A. Czaplewski, ${ }^{\mathrm{f}}$ Mengyao Dong, ${ }^{\mathrm{g}, \mathrm{h}}$ Qian Shao, ${ }^{\mathrm{j}}$ Shide Wu, ${ }^{\mathrm{k}}$ Zhanhu Guo, ${ }^{\mathrm{h},{ }^{*}}$ and Jong E. Ryu ${ }^{\mathrm{a}, \mathrm{c},{ }^{*}}$

\footnotetext{
${ }^{a}$ Department of Mechanical Engineering, Indiana University-Purdue University Indianapolis, 799 W. Michigan St., Indidnapolis, IN 46202, USA

${ }^{\mathrm{b}}$ Division of Industrial Metrology, Korea Research Institute of Standards and Science, 267 Gajeong-ro, Yuseong-gu, Daejeon 34113, Korea

${ }^{\mathrm{c}}$ Composites Manufacturing Laboratory (CML), Department of Mechanical and Aerospace Engineering, North Carolina State University, 911 Oval Drive, Raleigh, NC 27695, USA

${ }^{\mathrm{d}}$ Materials and Manufacturing directorate, Air Force Research Laboratory, WPAFB, OH 45433, USA

${ }^{\mathrm{e}}$ Department of Electrical Engineering and Computer Science, Vanderbilt University, 2301 Vanderbilt Pl, Nashville, TN 37235, USA

${ }_{\mathrm{f}}^{\mathrm{f}}$ Center for Nanoscale Materials, Argonne National Laboratory, 9700 S. Cass Avenue Lemont, IL 60439, USA

g Key Laboratory of Materials Processing and Mold, Ministry of Education; National Engineering Research Center for Advanced Polymer Processing Technology, Zhengzhou University, Zhengzhou, 450002, China

${ }^{\mathrm{h}}$ Integrated Composites Laboratory (ICL), Department of Chemical \& Biomolecular Engineering, University of Tennessee, Knoxville, TN 37996, USA

i Division of Industrial Metrology, Korea Research Institute of Standards and Science, 267 Gajeong-ro, Yuseong-gu, Daejeon 34113, Korea

${ }^{j}$ College of Chemical and Environmental Engineering, Shandong University of Science and Technology, Qingdao, Shandong, 266590, China

${ }^{\mathrm{k}}$ Henan Provincial Key Laboratory of Surface and Interface Science, Zhengzhou University of Light Industry, No. 136, Science Avenue, Zhengzhou, 450001, China
}

\section{*Corresponding author:}

E-mail: jryu@ncsu.edu (J. R.); augustine.urbas@us.af.mil (A. U.); zahyun.ku.1.ctr@us.af.mil (Z. K.); and zguo10@utk.edu (Z.G.)

†These authors contributed equally.

This is the author's manuscript of the article published in final edited form as:

Berndt, A. J., Hwang, J., Islam, M. D., Sihn, A., Urbas, A. M., Ku, Z., ... Ryu, J. E. (2019). Poly(sulfur-random-(1,3diisopropenylbenzene)) based mid-wavelength infrared polarizer: Optical property experimental and theoretical analysis. Polymer, 176, 118-126. https://doi.org/10.1016/j.polymer.2019.05.036 


\begin{abstract}
Development of polymer based mid-wavelength infrared (MWIR) optics has been limited mainly due to high optical loss of organic polymers used in general optical components. In this study, a MWIR polarization grating based on a sulfuric polymer poly(sulfur-random-(1,3-diisopropenylbenzene)) with a low loss in the MWIR range was fabricated using a simple two-step process: imprint and metal deposition. Fourier-transform infrared (FTIR) spectroscopy measurement showed that this polymeric MWIR polarizer selectively transmitted the polarized IR in transverse magnetic (TM) mode over the transverse electric (TE) mode at normal incidence. The measured extinction ratios $(\eta=$ The ratio of transmissions in TM and TE) were 208, 176, and 212 at the wavelength of 3, 4, and $5 \mu \mathrm{m}$, respectively. The computational simulation and analytical model confirmed that the enhanced TM transmission efficiency and $\eta$ followed a Fabry-Pérot (FP) resonance mode within the created sulfuric polymer film. This polymeric MWIR polarizer demonstrated a great potential for broader applications in IR photonics to realize low-cost and durable optical components.
\end{abstract}

KEYWORDS: sulfur polymer; Fabry-Pérot resonance; polarizer. 


\section{Graphical Abstract}

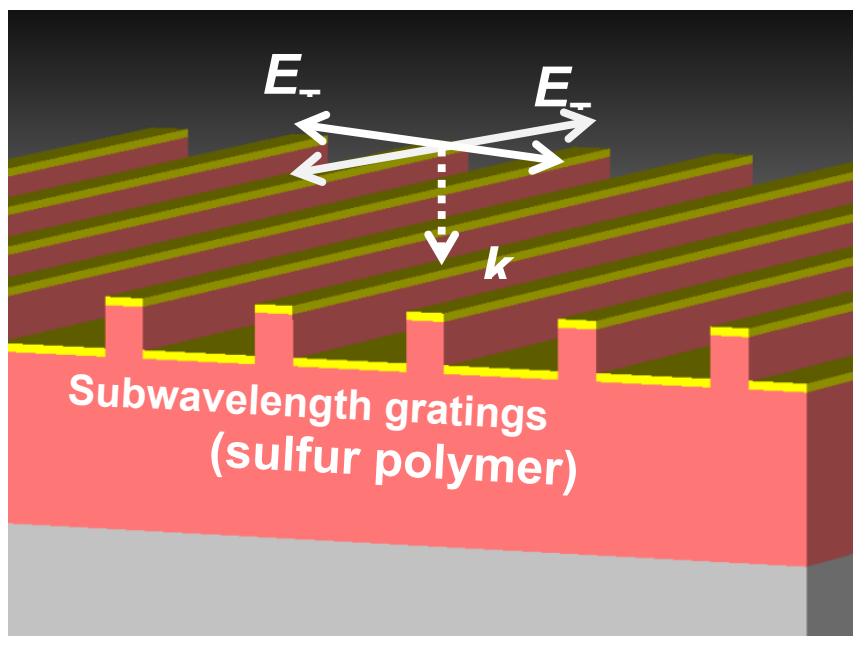

Low-cost Scalable Manufacturing Polymeric Mid-wavelength Polarizer

\section{Imprint!}

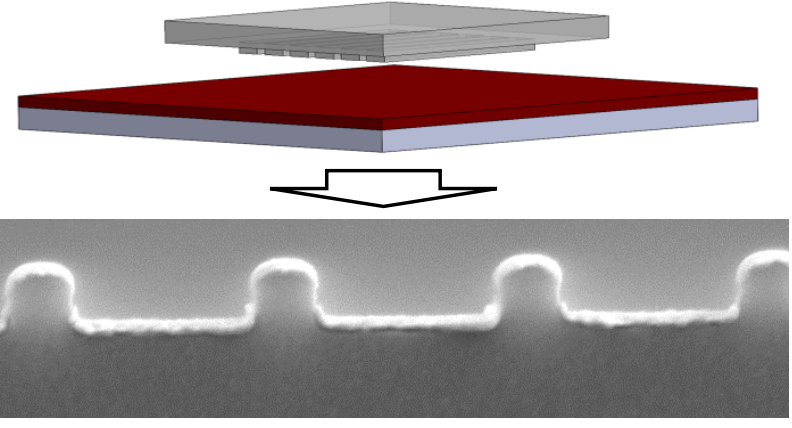




\section{Introduction}

Mid-wavelength infrared (MWIR, 3-5 $\mu \mathrm{m}$ ) sensors are widely used for a variety of applications including non-invasive medical diagnostics, gas detection, night vision, and thermal track and search [1-4]. In particular, there have been growing interests in the polarimetric imaging for MWIR because the visualization of polarization provides additional information such as surface feature, shading, and roughness. Further, the IR polarimetry has potential to distinguish radiation from synthetic objects surrounded by complex natural backgrounds, i.e. camouflage [5-8]. Due to the fast response and high sensitivity, state-of-the-art MWIR sensors are based on photon detectors such as $\mathrm{HgCdTe}$, InSb, quantum-well, quantum-dot, and type-II superlattice based IR photodetectors [9-11]. Inherently, those photodetectors lack the wavelength and polarization selectivity. In order to detect MWIR signal with a linear polarization characteristic, a wire-grid polarizer has been used in the IR sensor systems. For the MWIR wire-grid polarizer fabrication, two methods have been generally applied. First, a linear wire-grid is formed by mechanically ruling the MWIR transparent substrates and subsequently depositing conductive film (e.g., aluminum) at an oblique angle to generate parallel conductive wires at the peaks of the ruled grooves $[12,13]$. In the second technique, the wiregrid polarizers are fabricated by etching a pre-coated conductive film on the MWIR transparent substrate with a patterned photoresist mask, which is generated by the nanolithography technologies such as the interference lithography (IL) [14] and the nanoimprint lithography (NIL) [15-17]. In common, those commercial MWIR polarizers are fabricated on inorganic materials such as $\mathrm{BaF}_{2}, \mathrm{CaF}_{2}, \mathrm{KRS}-5, \mathrm{ZnS}$, and $\mathrm{ZnSe}$, which possess high refractive index (RI) and high transmission in the MWIR regime [18]. However, they are intrinsically expensive, brittle, and costly to manufacture into optical components including the polarizers [19-22].

Although the development of polymer-based MWIR optics has been challenged due to the low RI and high optical loss of organic materials [22], there have been growing interests in developing MWIR optical polymers to take advantages of polymers including lightweight, mechanical impact strength, and low-cost manufacturing process compared with the inorganic materials. Of note, polycarbonate (PC) and poly(methyl methacrylate) (PMMA) have been adopted in the 'visible light' $(400 \mathrm{~nm} \leq \lambda \leq 700 \mathrm{~nm})$ applications replacing the conventional optical glasses due to those advantages [23-27].

Sulfur element based polymers have recently been synthesized and demonstrated high RI and MWIR transparency [22,28-32]. The sulfur-based copolymer consists of elemental sulfur chains interconnected with a crosslink agent 1,3-diisopropenylbenzene (DIB). The sulfur-organic copolymers showed good chemical and mechanical 
processability, and high MWIR transmission compared to the conventional polymer materials used in optics (e.g., PMMA and PC) since the sulfur-element backbone reduces the vibrational absorption caused by heteroatom-hydrogen bonds, such as $\mathrm{C}-\mathrm{H}$ and $\mathrm{O}-\mathrm{H}[25,26,33-35]$. However, this novel polymer has not been fabricated into a practical MWIR optical component yet.

In this paper, a polymeric MWIR linear polarizer based on the organically modified sulfur polymer, poly(sulfur-random-(1,3-diisopropenylbenzene)) (poly(S-r-DIB)), was fabricated for the first time through a simple two-step manufacturing process: imprint and deposition. The transmissions of the MWIR waves polarized in transverse magnetic (TM) and transverse electric (TE) modes were characterized by Fourier transform infrared spectroscopy (FTIR). The underlying mechanism of the sulfur-polymeric grating (SPG) polarizer was also theoretically analyzed with 3-dimensional (3D) full field simulations, a multiple-layer model, and the Fabry-Pérot (FP) cavity resonance. While the novelty of this study is the invention of polymer based MWIR polarizer, in-depth theoretical analysis on the optical characteristics and the error due to fabrication are presented.

\section{Materials and Methods}

\subsection{Sulfur polymeric MWIR grating fabrication}

The poly(S-r-DIB) (35 wt $\%$ S) was synthesized through a method previously shown in the literatures $[22,28$ 32]. Elemental sulfur (Sigma Aldrich) was used without further treatment. Sulfur in the powder form was melt in an oil bath at $185^{\circ} \mathrm{C}$. The color and viscosity changes (yellow to red) were caused by the formation of an anion species during the ring opening of elemental sulfur [36]. A crosslinking agent, 1,3-diisopropenylbenzene (DIB, Sigma Aldrich), was measured and added to molten sulfur. The mixture was stirred by magnetic stirring bar until it became viscous. Then, the mixture was placed in an oven for $15 \mathrm{~min}$ at $175^{\circ} \mathrm{C}$ to complete the reaction. Air-cooled and solidified copolymer was dissolved in 1,2-dichlorobenzene (DCB, ACROS Organics) with a $1 \mathrm{~g} / \mathrm{mL}$ concentration for further coating processes. This concentration determined the thickness of spin-coated film.

Subwavelength polarizer gratings were imprinted on the poly(S-r-DIB) film by imprinting a polydimethylsiloxane (PDMS, Sylgard 184, Dow Corning) mold. A silicon master mold for the PDMS replication was fabricated by the electron beam lithography (EBL) and reactive ion etching (RIE) of the silicon wafer. The pitch, width, and height of the gratings in the silicon master mold were $1 \mu \mathrm{m}, 300 \mathrm{~nm}$, and $300 \mathrm{~nm}$, respectively. Degassed PDMS resin was cast on the silicon master mold and cured at $65^{\circ} \mathrm{C}$ for overnight. The poly(S-r-DIB) solution was spin-coated on a 300- $\mu \mathrm{m}$ thick double side polished (DSP) silicon wafer. The thin film of poly(S-r-DIB) was imprinted 
with the PDMS mold at 7-10 psi pressure and $180{ }^{\circ} \mathrm{C}$ for $1 \mathrm{hr}$. Finally, the PDMS mold was removed from the cooled wafer, and the $\mathrm{Cr} / \mathrm{Au}(5 \mathrm{~nm} / 50 \mathrm{~nm})$ was deposited by an electron beam evaporator with $0.5 \AA / \mathrm{s}$ rate. Both PDMS mold and silicon master mold were treated with a hydrophobic self-assembled molecule layer of $1 \mathrm{H}, 1 \mathrm{H}, 2 \mathrm{H}, 2 \mathrm{H}-$ perfluorooctyl-trichlorosilane $\left(\mathrm{CF}_{3}\left(\mathrm{CF}_{2}\right)_{5} \mathrm{CH}_{2} \mathrm{CH}_{2} \mathrm{SiCl}_{3}\right.$, Sigma Aldrich).

\subsection{Measurement and Simulation}

$50 \mu \mathrm{m}$ of poly(S-r-DIB) film on the DSP silicon wafer was used for the transmission measurement with Fourier-transform infrared spectroscopy (FTIR, ThermoFisher Scientific, Nicolet iS50) with a resolution of $2 \mathrm{~cm}^{-1}$ using a nitrogen-cooled mercury-cadmium-telluride detector. Holographic wire-grid polarizer with ZnSe substrate material was used for linear polarization of the incident light to the samples. FTIR measurements were taken at normal incidence. The cross-sections of Au-SPG were characterized with the scanning electron microscope (SEM, JEOL 7800F). Numerical simulations using CST Microwave Studio and COMSOL Multiphysics, which use the finite integration technique (FIT) [37,38] and the finite element method (FEM) [39-41], respectively, were carried out to obtain the solution of Maxwell's equation. In the simulation, $n_{S i}=3.4$ and $n_{S P G}=1.9$ were used for the refractive indices for silicon and SPG, respectively. The Au thin-film was described by the Drude model with plasma frequency $\left(\omega_{\mathrm{p}}=9.03 \mathrm{eV}\right)$ and collision frequency $\left(\omega_{c}=0.038 \mathrm{eV}\right)[42]$

\section{Results and Discussion}

The poly(S-r-DIB) fabricated for this study contains $35 \mathrm{wt} \%$ of sulfur, and the bare film (thickness: $50 \mu \mathrm{m}$ ) shows high FTIR transmittance in MWIR (Fig. 1). High transmission of the poly(S-r-DIB) film in the MWIR proves its feasibility as a polymeric substrate material of MWIR optics such as the wire-grid polarizers. 
(a)

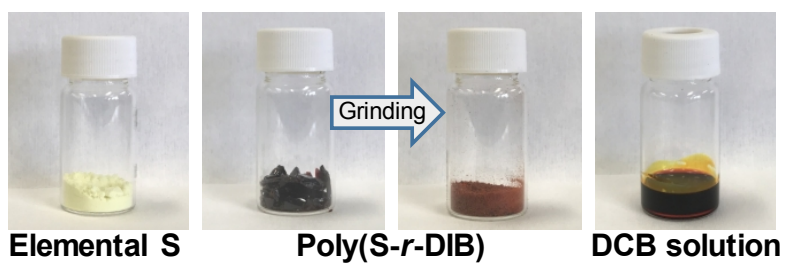

(b)

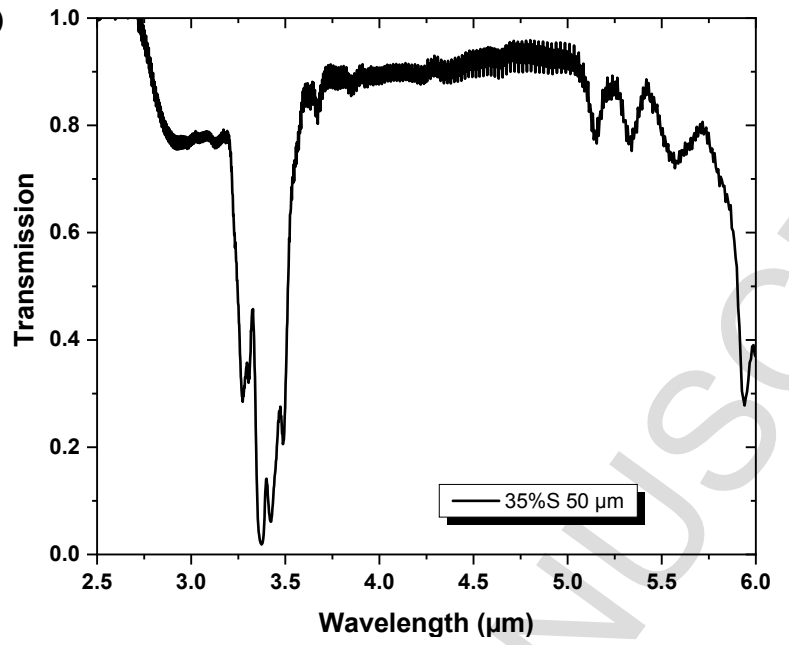

Fig. 1. Synthesis of poly(S-r-DIB) solution for micro/nanofabrication. (a) Elemental sulfur to the poly(S-r-DIB) solution in DCB. (b) Transmission spectra of poly(S-r-DIB) film (35 wt $\% \mathrm{~S})$ on silicon substrate.

Various configurations of Au-SPG plasmonic polarizers were considered in the design stage. The tested grating structures noted as 'A', 'B', 'C,' and 'D' are shown in Fig. 2. The simulation of the polarization performance was conducted with CST Microwave Studio for the variety of structure configurations.
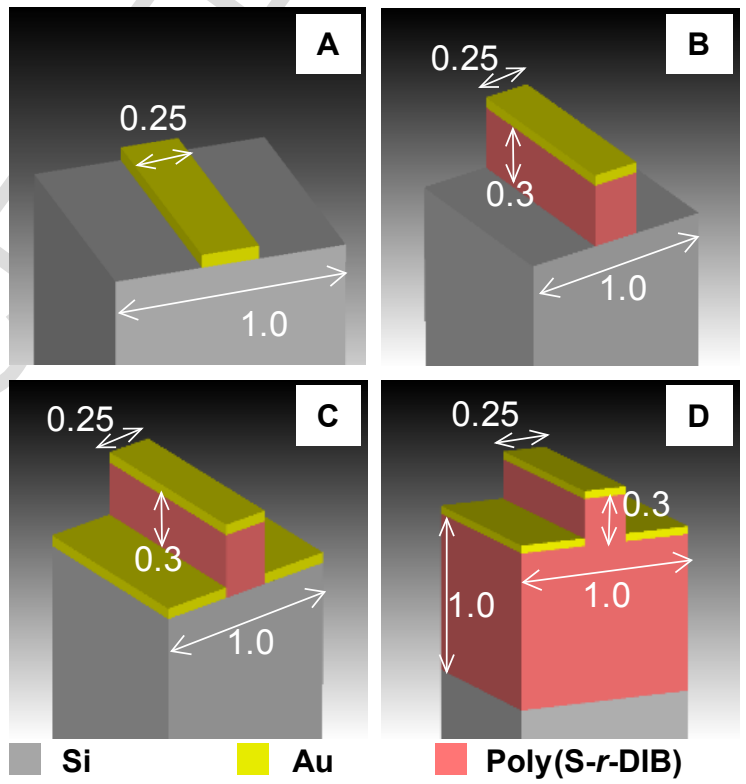
Fig. 2. Au grating configurations (A-D) with various poly(S-r-DIB) structures. Dimensions used in the computational simulation are shown (unit: $\mu \mathrm{m}$ ). This thickness of Au is $50 \mathrm{~nm}$.

In Fig. 3, the configurations A and C have a similar low TM transmission performance with the dip at 3.4 $\mu \mathrm{m}$ due to the surface plasmon (SP) resonance at the interface of Au and Si substrate. This dip is reflected in the extinction ratio ( $\eta=$ the ratio of transmissions in TM and TE). On the other hand, the Au grating on the poly(S- $r$ DIB) fins in the configuration C reduces TE transmission, and therefore enhances the extinction ratio ( $\eta$ ) compared with the configuration A. Comparing configurations B and C, the B shows a higher transmissions in TE mode, which result in a lower extinction ratio $(\eta<10)$ across the wavelength range of interest. The $\mathrm{C}$ has Au grating layers both on the Si substrate as well as on the poly(S-r-DIB) fins, while the B has Au gratings only on the poly(S-r-DIB) fins. Higher TM transmission and lower TE transmission of the configuration C (Fig. 3a) is resulted from the surface plasmon resonance at the $\mathrm{Au}$ and $\mathrm{Si}$ interface, which is absent in the configuration $\mathrm{B}$. Unlike other three configurations, the configuration D has the poly(S-r-DIB) thin film between the Au gratings and silicon substrate, which results in a distinctive TM transmission peak at $3.6 \mu \mathrm{m}$ and the lowest TE transmission among the tested samples. The transmissions in the $1^{\text {st }}$ order diffraction, $T+/-1$, were estimated with COMSOL Multiphysics, and the use of COMSOL was validated by comparing the $0^{\text {th }}$ order diffraction transmissions with the CST Microwave Studio (Fig. 3d). COMSOL (symbol) and CST (solid line) both show almost identical results for the $0^{\text {th }}$ order diffraction transmission $\left(T_{0}\right)$ (Fig. 3d). While the configurations $\mathrm{A}$ and $\mathrm{C}$ show a significant $1^{\text {st }}$ order diffraction in the shorter wavelength regime ( $\lambda<3.4 \mu \mathrm{m}$, the wavelength of first order SP resonance), the configurations B and D have a negligible $1^{\text {st }}$ order diffraction, which is a desirable characteristic as a polarizer. Based on the computational simulation, the configuration D, where the bilayer Au-SPGs are on the poly(S-r-DIB) thin film, was selected for the fabrication and demonstration due to the high TM transmission, high extinction ratio, and nearly zero $1^{\text {st }}$ diffraction order transmission. 

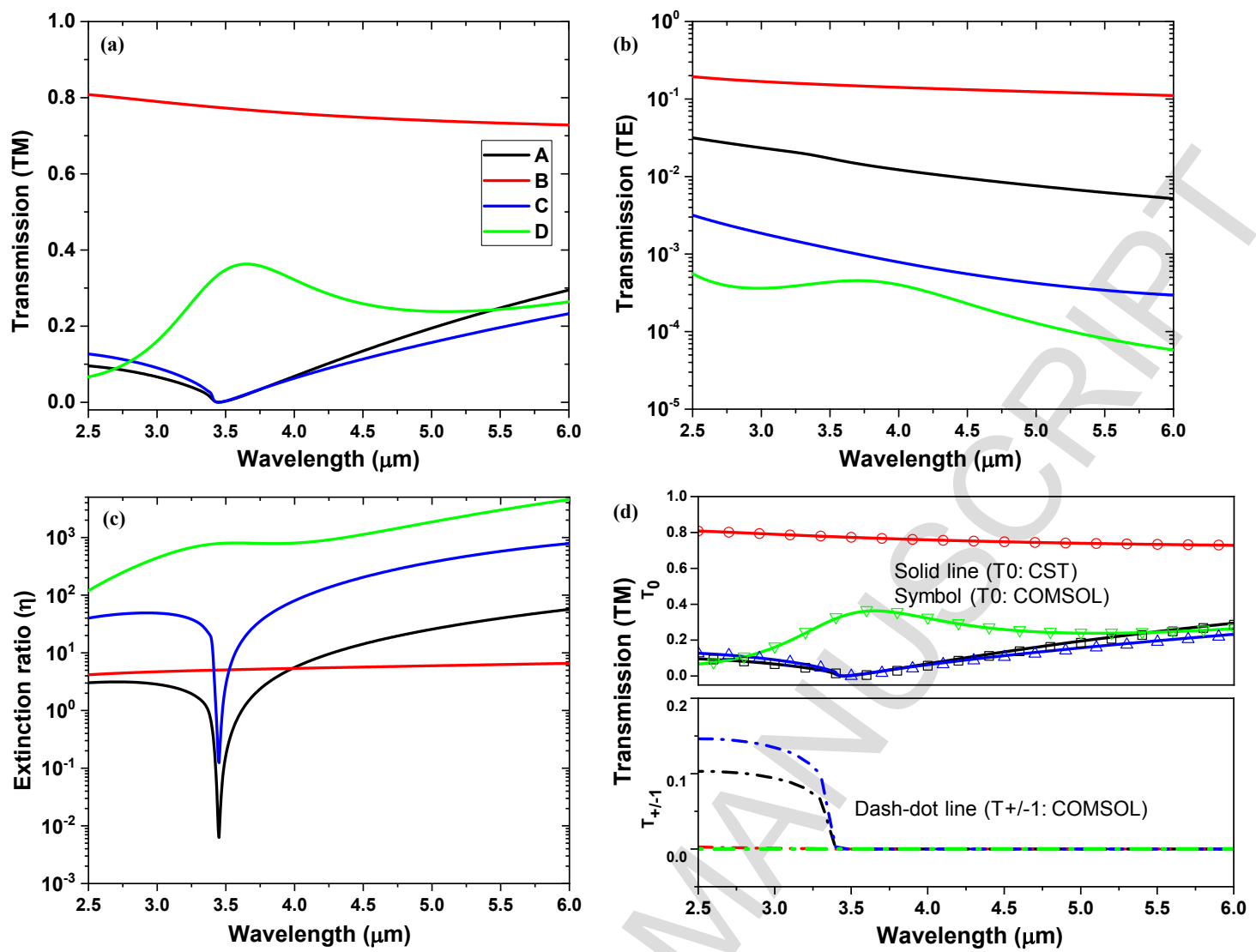

Fig. 3. Four grating configurations and computational simulation of transmissions (Dimensions of A-D configurations are shown in Fig. 2). (a) TM transmission. (b) TE transmission. (c) Extinction ratio ( $\eta$ ). (d) The $0^{\text {th }}$ order diffraction $\left(T_{0}\right.$ ) calculated by CST and COMSOL (top) and the $1^{\text {st }}$ order diffraction transmission $\left(T_{+/-1}\right)$ estimated with COMSOL.

Fig. 4 illustrates the fabrication processes and results of the bilayer Au-SPG with the thin poly(S-r-DIB) film on silicon substrate. In brief, spin coated poly(S-r-DIB) film in the target thickness was imprinted with a PDMS stamp at an elevated temperature and pressure. Bilayer Au gratings were obtained by subsequently depositing the $\mathrm{Cr} / \mathrm{Au}$ film on the imprinted SPG. The cross-sections of nanoimprinted poly(S-r-DIB) film before and after the $\mathrm{Cr} / \mathrm{Au}$ deposition are shown in the scanning electron micrographs (Fig. 4d and 4e). Designed pitch ( $p$ ), grating width ( $w$ ), grating height $\left(t_{s g}\right)$, and poly(S-r-DIB) thickness $\left(t_{s f}\right)$, and Au thickness $\left(t_{A u}\right)$ for this experiment were $1000 \mathrm{~nm}, 250 \mathrm{~nm}, 300 \mathrm{~nm}$, $1000 \mathrm{~nm}$, and $50 \mathrm{~nm}$, respectively. While the intended design was a rectangular cross-section, the actual imprinted gratings had round edges and angled-sidewalls. The factors affecting nanoimprint profile include the polymer thickness, imprint pressure, mold aspect ratio, and thermodynamic characteristics of the polymer [43]. If accurate 
grating shape is a critical factor for the performance of the plasmonic polarizer, a comprehensive design optimization process with the mold shape (e.g., pitch, grating height, etc.), process parameters (e.g., imprinting pressure, temperature, time, polymer thickness, etc.), and material properties (e.g., sulfur content, PDMS mold stiffness, etc.), will be required as a subsequent study. Here, the effect of actual imprint profile on the polarizer performance is analyzed in the following sections.

(a)

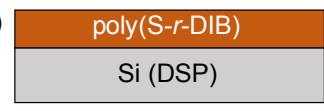

(b)

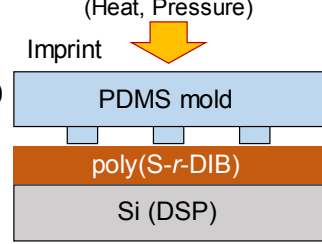

(c)

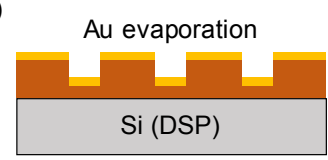

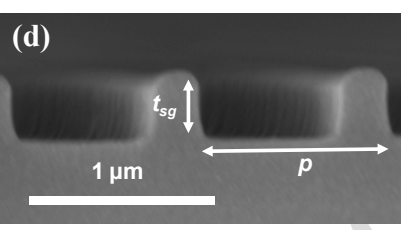

(e)

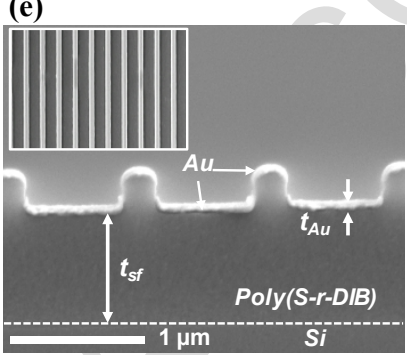

Fig. 4. Fabrication of bilayer Au grating on the sulfuric polymer. (a) Spin coating poly(S-r-DIB) on a DSP silicon wafer. (b) Thermal nanoimprint lithography (NIL) with the PDMS mold. (c) Deposition of $\mathrm{Cr} / \mathrm{Au}(5 \mathrm{~nm} / 50 \mathrm{~nm})$ on the imprinted substrate. (d) Cross-section SEMs after NIL and (e) after metal film deposition (inset: the top-view of the Au gratings).

The transmissions of linearly polarized MWIR through the bilayer Au-SPG were measured by the FTIR. All FTIR measurements were taken at normal incidence to the samples. The transmission spectra for TM and TE, and the extinction ratio $(\eta)$ for the bilayer Au-SPG are shown in Fig. 5. The transmissions of TM (TE) polarized MWIR are $0.25,0.31$, and $0.24(0.0012,0.0018$, and 0.0011$)$ at a wavelength of 3,4 , and $5 \mu \mathrm{m}$, respectively. Therefore, the extinction ratios at $3 \mu \mathrm{m}, 4 \mu \mathrm{m}$, and $5 \mu \mathrm{m}$ of wavelengths are calculated to be 208, 176, and 212, respectively (Fig. 5b). The overall trend of measured TM transmission agrees well with the simulation (CST Microwave Studio) result while the TE transmissions show larger disparity (Fig. 5c). Measured TE transmission showed relatively small changes across the wavelength. However, the TE transmission in simulation consistently decreases from $5 \times 10^{-4}$ to 5 $\times 10^{-5}$ in the $4 \mu \mathrm{m}$ and $6 \mu \mathrm{m}$ range. This decrease in TE transmission results in upward trend in the extinction ratio at 
the longer wavelength than $4 \mu \mathrm{m}$ (Fig. 5d). The discrepancy in transmission intensities may be induced by the imperfections and errors in the fabrication that were mentioned above.
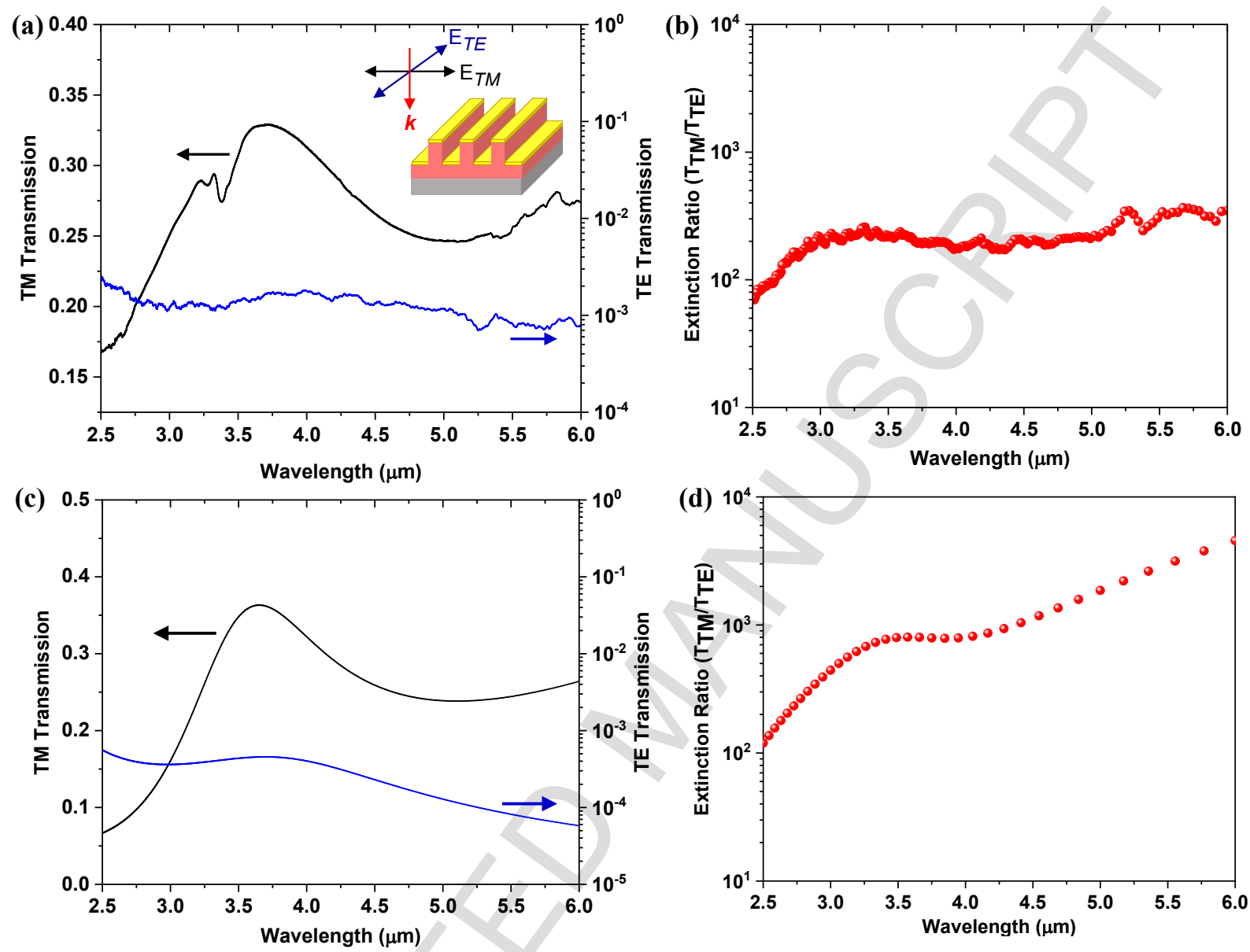

Fig. 5. (a) FTIR-measured transmission (black: TM, blue: TE). TM- and TE-directions are illustrated in the inset. (b)

Extinction ratio calculated using the measurement results $\left(\eta=T_{T M} / T_{T E}\right)$. (c) CST-simulated transmission. (d) Extinction ratio obtained using the simulation results.

For a better understanding of the response of the bilayer Au-SPG film on silicon wafer, the transmission and reflection of the composite structure were analyzed with a multiple-layer model based on the transfer matrix method $[38,44-46]$. The overall transfer matrix $\left(M_{t o t}\right)$ can be obtained by multiplying the transfer matrix of each layer, $M_{t o t}=$ $M_{1} \cdot M_{2} \cdot M_{3}$, where $M_{1}, M_{2}$, and $M_{3}$ are the transfer matrices for the 1) air/Au SPG, 2) poly(S-r-DIB) film, and 3 ) poly(S-r-DIB)/silicon configurations, respectively [44]. Those transfer matrices are calculated by following procedure. 
The general transfer matrix described by the $S$-parameters is [47]:

$$
M=\left(\begin{array}{cc}
S_{12}-S_{11} S_{22} / S_{21} & S_{11} / S_{21} \\
-S_{22} / S_{21} & 1 / S_{21}
\end{array}\right)
$$

where $S_{i j}$ represents the response at port $i$ due to a signal at port $j$. Therefore, in this study, $S_{12}$ and $S_{21}$ are the transmission coefficients in forward and backward directions. Similarly, $S_{11}$ and $S_{22}$ are the reflection coefficients at the front and backsides of the interfaces, respectively. The reflection/transmission coefficients with corresponding $S$ parameters at each layer are illustrated in Fig. 6.

(a)

Air / Au-SPG

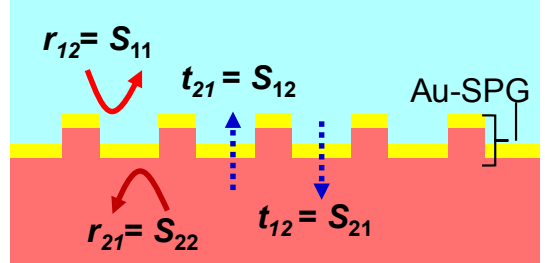

(b)

poly(S-r-DIB)

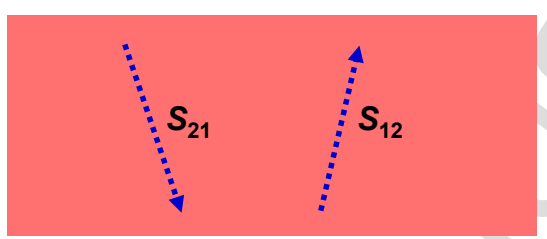

(c) $\quad \operatorname{poly}(S-r-D I B) / S i$

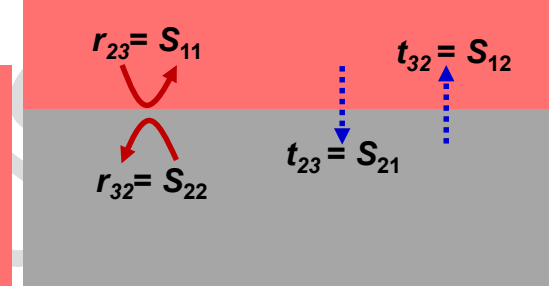

Fig. 6. Transmission and reflection coefficients in $S$-parameters in the decoupled structures corresponding to (a) $M_{1}$, (b) $M_{2}$, and (c) $M_{3}$ transfer matrices.

Therefore, the transfer matrix $M_{1}$ can be written as

$$
M_{1}=\left(\begin{array}{cc}
t_{21}-r_{12} r_{21} / t_{12} & r_{12 / t_{12}} \\
-r_{21} / t_{12} & 1 / t_{12}
\end{array}\right)
$$

where $r_{12}$ and $r_{21}$ are the reflection coefficients of the Au-SPG at the air and poly(S-r-DIB) film sides, respectively. $t_{12}$ and $t_{21}$ are the transmission coefficients for the electromagnetic wave propagating through the Au-SPG from air and from poly(S-r-DIB) film, respectively $[44,45,48]$. For $M_{2}$, the poly(S-r-DIB) layer was modeled to present only propagations of transmitted light. The phase of propagating wave was added by the propagating phase factor, $\beta=$ $n_{\text {poly }(\mathrm{S}-r-\mathrm{DIB})} \cdot k \cdot t_{s f}$ where $n_{\text {poly(S }-r-\mathrm{DIB})}, k$, and $t_{s f}$ are the refractive index of poly(S-r-DIB), the wave vector, and the thickness of the poly(S-r-DIB) layer, respectively. Therefore, the $S_{21}$ and $S_{12}$ in the poly(S-r-DIB) layer can be described by

$$
\begin{aligned}
& S_{21}=\frac{\tilde{t}_{12} e^{i \beta}}{\tilde{t}_{12}}=e^{i \beta} \\
& S_{12}=\frac{\tilde{t}_{32} e^{i \beta}}{\tilde{t}_{32}}=e^{i \beta}
\end{aligned}
$$

Since $S_{11}$ and $S_{22}$ are not considered in the poly(S-r-DIB) layer, the $M_{2}$ is reduced to 


$$
M_{2}=\left(\begin{array}{cc}
e^{i \beta} & 0 \\
0 & e^{-i \beta}
\end{array}\right)
$$

Similarly, $M_{3}$ can be written as follows:

$$
M_{3}=\left(\begin{array}{cc}
t_{32}-r_{23} r_{32} / t_{23} & r_{23} / t_{23} \\
-r_{32} / t_{23} & 1 / t_{23}
\end{array}\right)
$$

Where $r_{23}\left(t_{23}\right)$ and $r_{32}\left(t_{32}\right)$ are coefficient of reflection (transmission) at the interface for the wave propagating from the poly(S-r-DIB) and from the silicon substrate, respectively $[44,45,48]$.

The overall transfer matrix can be calculated to be

$$
\begin{aligned}
& M=M_{1} \cdot M_{2} \cdot M_{3}=\left(\begin{array}{cc}
t_{21}-r_{12} r_{21} / t_{12} & r_{12 / t_{12}} \\
-r_{21} / t_{12} & 1 / t_{12}
\end{array}\right) \cdot\left(\begin{array}{cc}
e^{i \beta} & 0 \\
0 & e^{-i \beta}
\end{array}\right) \cdot\left(\begin{array}{cc}
t_{32}-r_{23} r_{32} / t_{23} & r_{23} / t_{23} \\
-r_{32} / t_{23} & 1 / t_{23}
\end{array}\right) \\
& =\left(\begin{array}{cc}
\left(t_{21}-\frac{r_{12} r_{21}}{t_{12}}\right)\left(t_{32}-\frac{r_{23} r_{32}}{t_{23}}\right) e^{i \beta}-\left(\frac{r_{12} r_{32}}{t_{12} t_{23}}\right) e^{-i \beta} & \left(t_{21}-\frac{r_{12} r_{21}}{t_{12}}\right)\left(\frac{r_{23}}{t_{23}}\right) e^{i \beta}+\left(\frac{r_{12}}{t_{12} t_{23}}\right) e^{-i \beta} \\
-\left(\frac{r_{21}}{t_{12}}\right)\left(t_{32}-\frac{r_{23} r_{32}}{t_{23}}\right) e^{i \beta}-\frac{r_{32}}{t_{23} t_{12}} e^{-i \beta} & -\left(\frac{r_{21} r_{23}}{t_{12} t_{23}}\right) e^{i \beta}+\left(\frac{1}{t_{12} t_{23}}\right) e^{-i \beta}
\end{array}\right) \\
& =\left(\begin{array}{cc}
S_{12}-S_{11} S_{22} / S_{21} & S_{11} / S_{21} \\
-S_{22} / S_{21} & 1 / S_{21}
\end{array}\right)
\end{aligned}
$$

The overall reflection coefficient, $S_{11}$, can be calculated by dividing $S_{11 / S_{21}}$ with $1 / S_{21}$ in (7).

$$
S_{11}=r=\frac{S_{11} / S_{21}}{1 / S_{21}}=\frac{\left(t_{21}-\frac{r_{12} r_{21}}{t_{12}}\right)\left(\frac{r_{23}}{t_{23}}\right) e^{i \beta}+\left(\frac{r_{12}}{t_{12} t_{23}}\right) e^{-i \beta}}{-\left(\frac{r_{21} r_{23}}{t_{12} t_{23}}\right) e^{i \beta}+\left(\frac{1}{t_{12} t_{23}}\right) e^{-i \beta}}
$$

Similarly, the overall transmission coefficient, $S_{21}$, is

$$
S_{21}=t=\left(1 / S_{21}\right)^{-1}=\left(-\left(\frac{r_{21} r_{23}}{t_{12} t_{23}}\right) e^{i \beta}+\left(\frac{1}{t_{12} t_{23}}\right) e^{-i \beta}\right)^{-1}
$$

After algebraic simplification, (8) and (9) are expressed as

$$
r=\frac{r_{12}+\alpha r_{23} \exp (2 i \beta)}{1-r_{21} r_{23} \exp (2 i \beta)}, t=\frac{t_{12} t_{23} \exp (i \beta)}{1-r_{21} r_{23} \exp (2 i \beta)}
$$

where $\alpha$ is $t_{21} t_{12}-r_{21} r_{12} \approx 0$.

Each transmission and reflection coefficient $\left(t_{\mathrm{ij}}\right.$ and $\left.r_{\mathrm{ij}}\right)$ were obtained through numerical simulations (CST Microwave Studio) modeled with the only corresponding layers (i.e. air / Au-SPG model for $r_{12}, r_{21}, t_{12}, t_{21}$; poly(S-rDIB) / silicon substrate for $r_{23}, t_{23}$ ). In Fig. 7, the amplitude and phase of reflection and transmission coefficients of 
bilayer Au-SPG film based on the intended design (Fig. 7a and 7c) and the actually imprinted geometry (Fig. 7b and 7d) were evaluated and compared. The modified model to simulate the imprinted geometry is shown in Fig. 8 .
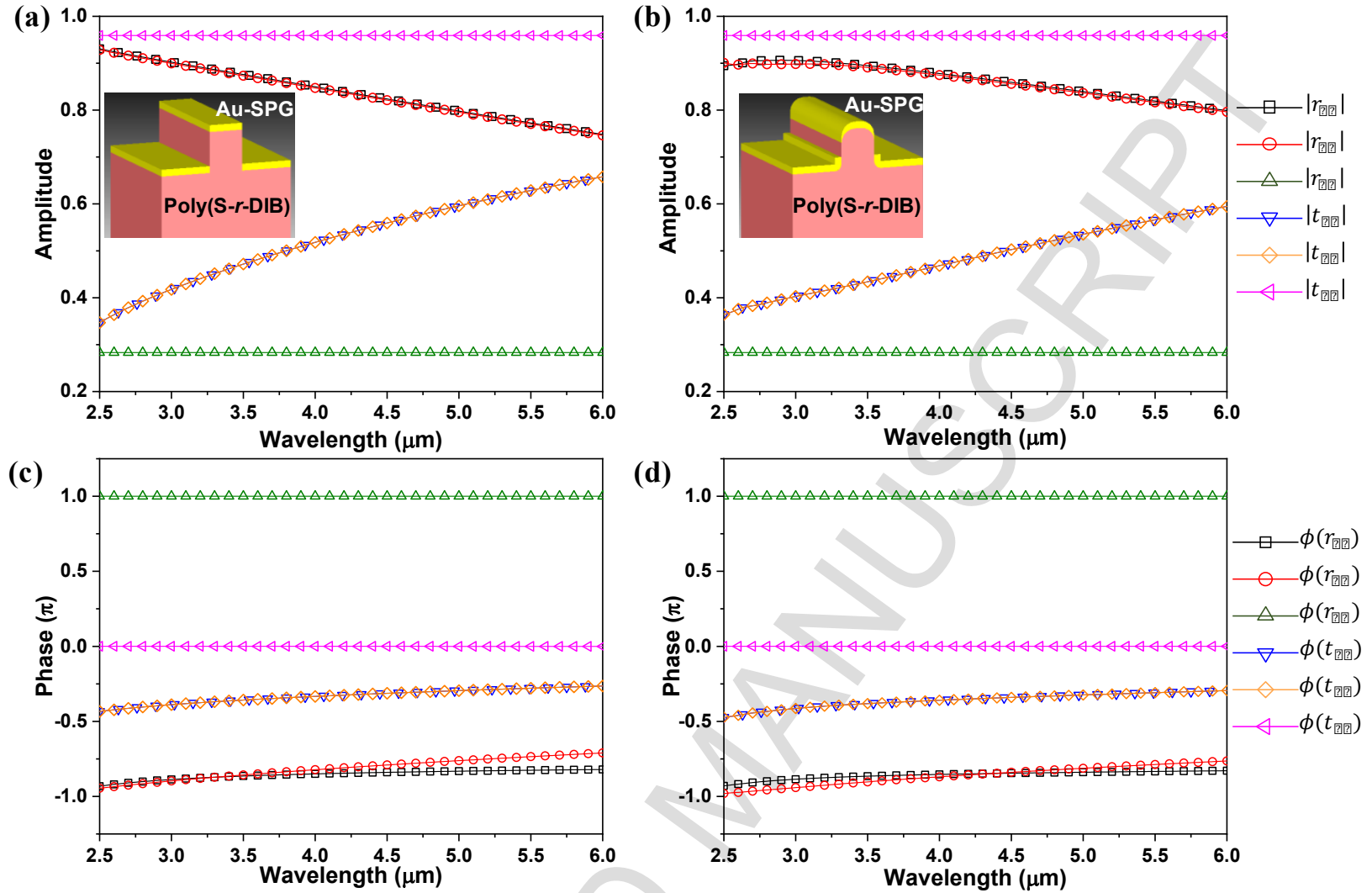

Fig. 7. Amplitude and phase terms of reflection and transmission coefficients obtained from the numerical simulations of $(\mathrm{a}, \mathrm{c})$ ideal-shaped bilayer Au-SPG film (designed gratings: rectangular cross-section without the sidewall deposition) and (b,d) fabrication-induced bilayer Au-SPG film. The geometric imperfections lead to slight differences only in the related parameters $\left(r_{12}, r_{21}, t_{12}\right.$, and $\left.t_{21}\right)$, whose decoupled configuration contains the bilayer Au-SPG at the interface (Fig. 6).

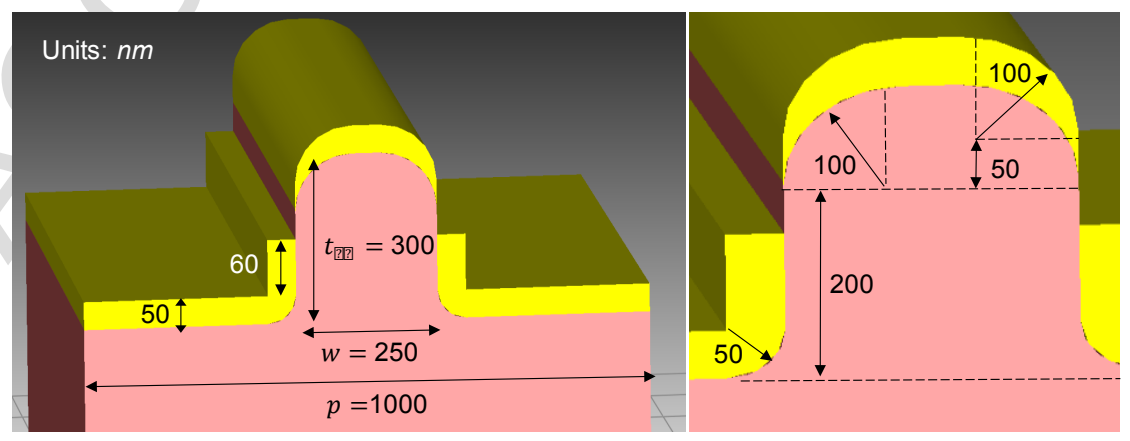


Fig. 8. Modified grating geometry for computational simulations of the actually fabricated bilayer Au-SPG.

The multiple-layer model based overall reflection and transmission, $R_{\text {cal }}=|r|^{2}$ and $T_{\text {cal }}=|t|^{2}$ in (10), match well with the CST simulation results $\left(R_{\text {sim }}\right.$ and $\left.T_{\text {sim }}\right)\left(\right.$ Fig. 9b). $T_{\text {cal }}$ also shows similar trends to the FTIR measured TM transmission shown in Fig. 5a. However, the peak locations of simulated and calculated transmission are found to be slightly shifted from the FTIR measurement. The peak in the FTIR measured TM transmission spectrum is at $3.7 \mu \mathrm{m}$ while the theoretical results $T_{\text {cal }}$ have peaks at $3.6 \mu \mathrm{m}$. This discrepancy may be due to the imperfection from the fabrication process.
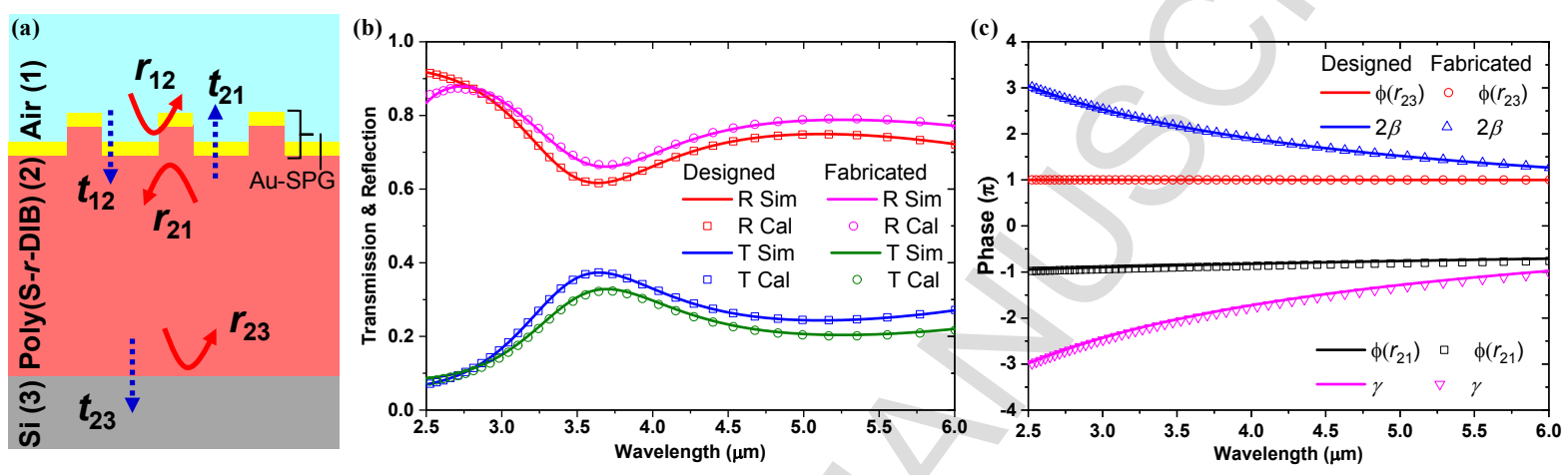

Fig. 9. (a) The multiple-layer model analysis. $r_{i j}$ and $t_{i j}$ are the reflection and transmission coefficients, respectively, where the subscripts $i, j$ indicate the layers. (b) Overall reflection $\left(R=|r|^{2}\right)$ and transmission $\left(T=|t|^{2}\right)$ obtained by the multiple-layer model (symbol) and CST simulation of the entire structure (solid line). The comparison was made based on the designed and the fabricated sample dimensions. (c) Phases $\phi\left(r_{21}\right), \phi\left(r_{23}\right), \gamma$, and $2 \beta$ used in the FP cavity resonance condition.

As shown in Fig. 4e, the SEM cross-section of polymer gratings is not rectangular, and the bottom Au film is curved up and deposited on the sidewall of gratings. Of note, the profile of nanoimprinted polymer is affected by process parameters including polymer thickness, aspect ratio of printed structure, and thermodynamic properties of polymer $[43,49]$. In this study, the significance of those fabrication imperfections caused by the materials and fabrication method was further investigated by the simulation and multiple-layer analysis based on a modified model reflecting the fabrication imperfection (Fig. 8). The comparison of $R$ and $T$ obtained from the designed model and fabricated model shown in Fig. 9b reveals that the overall characteristics are not much affected by the fabrication 
imperfection (i.e., the round grating edges and sidewall deposition). On the other hand, the transmission peak intensity is slightly diminished and shifted toward $3.7 \mu \mathrm{m}$, which is closer to the FTIR measured TM transmission.

Multiple reflections between two interfaces (Au-SPG / poly(S-r-DIB) film and poly(S-r-DIB) film / silicon substrate) can cause the TM transmission intensity to increase when the FP cavity condition is satisfied, i.e., the constructive interference inside poly(S-r-DIB) film is occurred by (11).

$$
\gamma=\phi\left(r_{21}\right)+\phi\left(r_{23}\right)-2 \beta=2 m \pi \text { for }|m|=0,1,2, \ldots
$$

where $\gamma$ is the round-trip propagation phase inside the poly(S-r-DIB) film and $\phi$ is the phase of the reflected waves at the interfaces. $\phi\left(r_{21}\right)$ and $\phi\left(r_{23}\right)$ were obtained with CST simulation with the models based on the dimensions of the designed and the fabricated samples (Fig. 9c). The FP resonance condition of $\gamma=-2 \pi$ is satisfied at $\lambda=3.5 \mu \mathrm{m}$ (designed sample) and $3.6 \mu \mathrm{m}$ (fabricated sample), which closely match with the location of the transmission peaks calculated with the simulation and multiple-layer model. This indicates that the enhanced transmission is mainly attributed to the FP cavity resonance inside poly(S-r-DIB) film, satisfying (11). Note that the peak wavelength of TM transmission is slightly shifted from FP resonance wavelength resulting from the coupling between SP and FP resonances [50]. As shown in Fig. 9c, $\gamma$ is predominantly controlled by the propagating phase factor $\beta$ since the phase changes in $r_{21}$ and $r_{23}$ are negligible along the wavelength. Therefore, the poly(S-r-DIB) thickness (i.e. $t_{s f}$ ) should be carefully tuned to obtain desired characteristics in the target wavelength realm (i.e., MWIR).

The calculated round-trip propagation phase $(\gamma)$, the simulated TM transmission and extinction ratio $(\eta)$ are color-mapped as a function of the poly(S-r-DIB) thickness $\left(t_{s f}\right)$ and wavelength $(\lambda)$ as shown in Fig. 10. The FP resonance conditions $(\gamma=-8 \pi$ to 0 with a step of $2 \pi)$ is indicated as dash lines in Fig. 10a and 10b, respectively. It is important to note that the dash lines of $\gamma$ (FP resonance) are overlapped well with the peak locations as indicated in the Fig. 10b in the wavelength range of 2-6 $\mu \mathrm{m}$. Fig. 10b and 10c provides the design guidance for the thickness of poly(S-r-DIB) to achieve high transmission efficiency and high extinction ratio at the target regime (e.g., MWIR, 3-5 $\mu \mathrm{m})$. According to this analysis, the first order FP cavity resonance $(\gamma=-2 \pi)$ at $t_{\text {poly }(\mathrm{S}-r-\mathrm{DIB})} \cong 1 \mu \mathrm{m}$ was the suitable design to realize the broadband polarizer for the MWIR region. 

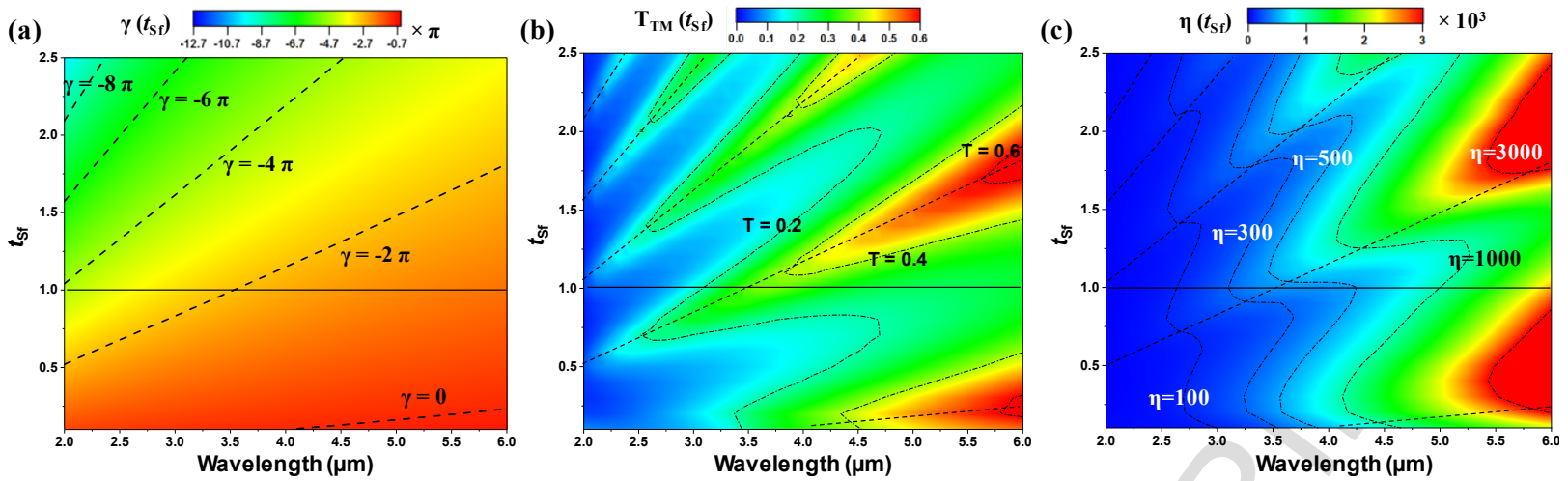

Fig. 10. Color maps of the round-trip propagation phase $(\gamma)$, transmission $(T)$, and extinction ratio $(\eta)$ for the TMpolarized incident light as a function of the poly(S-r-DIB) film thickness $t_{\mathrm{poly}}(\mathrm{S}-r-\mathrm{DIB})$ and the wavelength. (a) The dash lines indicate where the FP cavity resonance condition of $\gamma=-8 \pi \sim 0$ (with $2 \pi$ step) is satisfied. (b-c) Simulation of TM transmission $\left(T_{T M}\right)$ and extinction ratio $(\eta)$. The dash lines connect the local maxima with the given film thickness. Black horizontal line indicates $t_{s f}$ (thickness of poly(S-r-DIB) film) $=1 \mu \mathrm{m}$ in the experiment.

\section{Conclusion}

MWIR polarizer with high transmission efficiency and extinction ratio were successfully demonstrated for MWIR optics with the recently invented sulfur-based polymer material. Advantages of polymer manufacturing include the use of cost-efficient and scalable nanoimprint processes to fabricate the MWIR polarizers. FTIR-measured transmission of Au bilayer gratings fabricated on the poly(S-r-DIB) film showed a high extinction ratio in the MWIR regime (208 at $3 \mu \mathrm{m}, 176$ at $4 \mu \mathrm{m}$, and 212 at $5 \mu \mathrm{m}$ ). Through the computational simulations and the multiple-layer model, the enhanced transmission was found to be strongly related to the FP cavity resonance mode within the bilayer Au-SPG film. According to a higher extinction ratio means a better polarizer, the prepared polymer based MWIR polarizer achieved the extinction ratios about 200 , while the extinction ratio of commercial holographic wire-grid polarizers based on $\mathrm{BaF}_{2}, \mathrm{CaF}_{2}$, KRS-5 or ZnSe is generally 150-300 in the same wavelength region. This promising result allows us to expect that further design optimization study, which is a following research topic, will lead to the polymer-based MWIR polarizer with comparable or better optical performance than the current commercial products. Optimization of the polarizer will require the knowledge on the relationship between the transmission/reflection behavior of the Au-SPG and the design factors, including the grating pitch, width, poly(S-r-DIB) film thickness, and the height of SPG. This optimized polymer-based polarizer would significantly reduce the cost of IR sensing and 
imaging system while offering superior performance (i.e. high TM transmission and extinction ratio). Further, in order to apply the presented polymer-based MWIR polarizer in actual IR sensors, the technical challenges in manufacturing such as scaling up the grating area should be address in the following research. The study herein could also be broadly applied to develop other low-cost, durable, and characteristic adjustable MWIR optical components, such as bandpass filters, by using the sulfur-based polymer materials with different properties from traditional polymers or natural polymeric products [51-55].

\section{Acknowledgments}

J. Ryu was supported by the new faculty start-up fund at NCSU. This research was also supported in part by the Air Force Research Laboratory Materials and Manufacturing (RX) Directorate, through the Air Force Office of Scientific Research Summer Faculty Fellowship Program ${ }^{\circledR}$. The KRISS portion of this work was supported by Nano-Material Fundamental Technology Development Program (2018069993) through the National Research Foundation of Korea (NRF) funded by Ministry of Science and ICT, the KRISS grant GP2019-0015-03 and the AOARD grant FA238614-1-4094 funded by the U.S. government (AFOSR/AOARD). The AFRL portion of this work was supported by the AOARD grant FA2386-18-1-4104 funded by the U.S. government (AFOSR/AOARD). The Use of the Center for Nanoscale Materials at Argonne National Laboratory, an Office of Science user facility, was supported by the U.S. Department of Energy, Office of Science, Office of Basic Energy Sciences, under Contract No. DE-AC0206CH11357. We would like to acknowledge the Integrated Nanosystems Development Institute (INDI) in IUPUI for use of their JEOL SEM 7800F which was awarded through the NSF grant MRI-1229514.

\section{References}

[1] L. Hutchinson, Digital Infrared Breast Scan shows Promise for Detecting Cancer, Nature Rev. Clinical Oncology 7 (2010) 483.

[2] A. Rogalski, J. Antoszewski, L. Faraone, Third-Generation Infrared Photodetector Arrays, J. Appl. Phys. 105 (2009) 091101.

[3] T. Töpfer, K.P. Petrov, Y. Mine, D. Jundt, R.F. Curl, F.K. Tittel, Room-Temperature Mid-Infrared Laser Sensor for Trace Gas Detection, Appl. Optics 36 (1997) 8042-8049.

[4] Z. Han, P. Lin, V. Singh, L. Kimerling, J. Hu, K. Richardson, A. Agarwal, D. Tan, On-Chip Mid-Infrared Gas Detection using Chalcogenide Glass Waveguide, Appl. Phys. Lett. 108 (2016) 141106.

[5] M. Sarkar, D.S.S. Bello, C. van Hoof, A.J.P. Theuwissen, Biologically Inspired CMOS Image Sensor for Fast Motion and Polarization Detection, JSEN 13 (2013) 1065-1073. 
[6] J.S. Tyo, D.L. Goldstein, D.B. Chenault, J.A. Shaw, Review of Passive Imaging Polarimetry for Remote Sensing Applications, Appl. Optics 45 (2006) 5453-5469.

[7] A.G. Andreou, Z.K. Kalayjian, Polarization Imaging: Principles and Integrated Polarimeters, IEEE Sens. 2 (2002) 566-576.

[8] K.P. Gurton, A.J. Yuffa, G.W. Videen, Enhanced Facial Recognition for Thermal Imagery using Polarimetric Imaging, Optics Lett. 39 (2014) 3857-3859.

[9] A. Rogalski, Infrared Detectors: An Overview, Infrared Phys. Technol. 43 (2002) 187-210.

[10] A. Rogalski, Infrared Detectors: Status and Trends, Progress Quantum Electronics 27 (2003) 59-210.

[11] A. Rogalski, History of Infrared Detectors, Opto-Electron. Rev 20 (2012) 279-308.

[12] G.R. Bird, M. Parrish, The Wire Grid as a Near-Infrared Polarizer, JOSA 50 (1960) 886-891.

[13] J.B. Young, H.A. Graham, E.W. Peterson, Wire Grid Infrared Polarizer, Appl. Opt. 4 (1965) 1023-1026.

[14] I. Yamada, K. Kintaka, J. Nishii, S. Akioka, Y. Yamagishi, M. Saito, Mid-Infrared Wire-Grid Polarizer with Silicides, Optics Lett. 33 (2008) 258.

[15] S. Ahn, K. Lee, S.H. Lee, J. Kim, S.H. Kim, J. Park, P. Yoon, Fabrication of Subwavelength Aluminum Wire Grating using Nanoimprint Lithography and Reactive Ion Etching, Microelectronic Eng. 78 (2005) 314-318.

[16] S. Ahn, K. Lee, J. Kim, S.H. Kim, J. Park, S. Lee, P. Yoon, Fabrication of a 50 Nm Half-Pitch Wire Grid Polarizer using Nanoimprint Lithography, Nanotechnology 16 (2005) 1874-1877.

[17] G.P. Nordin, J.T. Meier, P.C. Deguzman, M.W. Jones, Micropolarizer Array for Infrared Imaging Polarimetry, JOSA A 16 (1999) 1168-1174.

[18] P. Miles, High Transparency Infrared Materials, Optical Eng. 15 (1976) 155451.

[19] X.H. Zhang, Y. Guimond, Y. Bellec, Production of Complex Chalcogenide Glass Optics by Molding for Thermal Imaging, J. Non-Crystalline Solids 326-327 (2003) 519-523.

[20] M. Aven, D. Marple, B. Segall, Some Electrical and Optical Properties of ZnSe, J. Appl. Phys. 32 (1961) 22612265.

[21] R. Kitamura, L. Pilon, M. Jonasz, Optical Constants of Silica Glass from Extreme Ultraviolet to Far Infrared at Near Room Temperature, Appl. Optics 46 (2007) 8118-8133.

[22] J.J. Griebel, S. Namnabat, E.T. Kim, R. Himmelhuber, D.H. Moronta, W.J. Chung, A.G. Simmonds, K. Kim, J. van der Laan, N.A. Nguyen, E.L. Dereniak, M.E. Mackay, K. Char, R.S. Glass, R.A. Norwood, J. Pyun, New Infrared Transmitting Material Via Inverse Vulcanization of Elemental Sulfur to Prepare High Refractive Index Polymers, Adv. Mater. 26 (2014) 3014-3018.

[23] L. Eldada, L.W. Shacklette, Advances in Polymer Integrated Optics, JSTQE 6 (2000) 54-68.

[24] C. Lü, B. Yang, High Refractive Index Organic-Inorganic Nanocomposites: Design, Synthesis and Application, J. Mater. Chem. 19 (2009) 2884-2901. 
[25] J. Liu, M. Ueda, High Refractive Index Polymers: Fundamental Research and Practical Applications, J. Mater. Chem. 19 (2015) 8907-8919.

[26] R. Okutsu, S. Ando, M. Ueda, Sulfur-Containing Poly(Meth)Acrylates with High Refractive Indices and High Abbe's Numbers, Chem. Mater. 20 (2008) 4017-4023.

[27] Z. Cui, C. Lü, H. Yang, B. Yang, J. Shen, X. Su, The Research on Syntheses and Properties of Novel Epoxy/Polymercaptan Curing Optical Resins with High Refractive Indices, Polymer 42 (2001) 10095-10100.

[28] L.E. Anderson, T.S. Kleine, Y. Zhang, D.D. Phan, S. Namnabat, E.A. LaVilla, K.M. Konopka, L. Ruiz Diaz, M.S. Manchester, J. Schwiegerling, R.S. Glass, M.E. Mackay, K. Char, R.A. Norwood, J. Pyun, Chalcogenide Hybrid Inorganic/Organic Polymers: Ultrahigh Refractive Index Polymers for Infrared Imaging, ACS Macro Lett. 6 (2017) 500-504.

[29] W.J. Chung, J.J. Griebel, E.T. Kim, H. Yoon, A.G. Simmonds, H.J. Ji, P.T. Dirlam, R.S. Glass, J.J. Wie, N.A. Nguyen, B.W. Guralnick, J. Park, A. Somogyi, P. Theato, M.E. Mackay, Y. Sung, K. Char, J. Pyun, The use of Elemental Sulfur as an Alternative Feedstock for Polymeric Materials, Nature Chem. 5 (2013) 518-524.

[30] J.J. Griebel, N.A. Nguyen, A.V. Astashkin, R.S. Glass, M.E. Mackay, K. Char, J. Pyun, Preparation of Dynamic Covalent Polymers Via Inverse Vulcanization of Elemental Sulfur, ACS Macro Lett. 3 (2014) 1258-1261.

[31] D.A. Boyd, C.C. Baker, J.D. Myers, V.Q. Nguyen, G.A. Drake, C.C. McClain, F.H. Kung, S.R. Bowman, W. Kim, J.S. Sanghera, ORMOCHALCs: Organically Modified Chalcogenide Polymers for Infrared Optics, Chem. Commun. 53 (2017) 259-262.

[32] D.A. Boyd, V.Q. Nguyen, C.C. McClain, F.H. Kung, C.C. Baker, J.D. Myers, M.P. Hunt, W. Kim, J.S. Sanghera, Optical Properties of a Sulfur-Rich Organically Modified Chalcogenide Polymer Synthesized Via Inverse Vulcanization and Containing an Organometallic Comonomer, ACS Macro Lett. 8 (2019) 113-116.

[33] R.A. Minns, R.A. Gaudiana, Design and Synthesis of High Refractive Index Polymers. II, J. Macromol. Sci.Pure Appli. Chem. 29 (1992) 19-30.

[34] M.A. Olshavsky, H.R. Allcock, Polyphosphazenes with High Refractive Indices: Synthesis, Characterization, and Optical Properties, Macromolecules 28 (1995) 6188-6197.

[35] C.A. Terraza, J. Liu, Y. Nakamura, Y. Shibasaki, S. Ando, M. Ueda, Synthesis and Properties of Highly Refractive Polyimides Derived from Fluorene-Bridged Sulfur-Containing Dianhydrides and Diamines, J. Polym. Sci. Part A: Polym. Chem. 46 (2008) 1510-1520.

[36] B. Meyer, Elemental Sulfur, Chem. Rev. 76 (1976) 367-388.

[37] CST Microwave Studio: A Numerical Simualtion Sofware for Electromagnetic Computing, Computer Simulation Technology GmbH, Darmstadt, Germany. Https://Www.Cst.Com/.

[38] J. Hwang, B. Oh, Y. Kim, S. Silva, J.O. Kim, D.A. Czaplewski, J.E. Ryu, E.K. Kim, A. Urbas, J. Zhou, Z. Ku, S.J. Lee, Fabry-Perot Cavity Resonance Enabling Highly Polarization-Sensitive Double-Layer Gold Grating, Scie. Rep. 8 (2018) 1-8.

[39] COMSOL Multiphysics, COMSOL AB, Stockholm, Sweden, Www.Comsol.Com.

[40] M. Ramezani, A. Halpin, A.I. Fernández-Domínguez, J. Feist, S.R. Rodriguez, F.J. Garcia-Vidal, J.G. Rivas, Plasmon-Exciton-Polariton Lasing, Optica 4 (2017) 31-37. 
[41] J. Smajic, C. Hafner, L. Raguin, K. Tavzarashvili, M. Mishrikey, Comparison of Numerical Methods for the Analysis of Plasmonic Structures, J. Computational Theoretical Nanoscie. 6 (2009) 763-774.

[42] M.A. Ordal, R.J. Bell, J. Alexander R W, L.L. Long, M.R. Querry, Optical Properties of Fourteen Metals in the Infrared and Far Infrared: Al, Co, Cu, Au, Fe, Pb, Mo, Ni, Pd, Pt, Ag, Ti, V, and W, Appl. Optics 24 (1985) 44934499.

[43] R.M. Al-Assaad, S. Regonda, L. Tao, S.W. Pang, W. Hu, Characterizing Nanoimprint Profile Shape and Polymer Flow Behavior using Visible Light Angular Scatterometry, J. Vacuum Sci. Technol. B: Microelectronics Nanometer Structures 25 (2007) 2396-2401.

[44] K. Bhattarai, Z. Ku, S. Silva, J. Jeon, J.O. Kim, S.J. Lee, A. Urbas, J. Zhou, A Large-Area, Mushroom-Capped Plasmonic Perfect Absorber: Refractive Index Sensing and Fabry-Perot Cavity Mechanism, Adv. Optical Mater. 3 (2015) 1779-1786.

[45] K. Bhattarai, S. Silva, K. Song, A. Urbas, S.J. Lee, Z. Ku, J. Zhou, Metamaterial Perfect Absorber Analyzed by a Meta-Cavity Model Consisting of Multilayer Metasurfaces, Sci. Rep. 7 (2017) 10569-9.

[46] H. Chen, J. Zhou, J.F. O'Hara, F. Chen, A.K. Azad, A.J. Taylor, Antireflection Coating using Metamaterials and Identification of its Mechanism, Phys. Rev. Lett. 105 (2010) 073901.

[47] J. Zhou, H. Chen, T. Koschny, A.K. Azad, A.J. Taylor, C.M. Soukoulis, J.F. O'Hara, Application of Metasurface Description for Multilayered Metamaterials and an Alternative Theory for Metamaterial Perfect Absorber, arXiv preprint arXiv:1111.0343 (2011) .

[48] J. Jeon, K. Bhattarai, D. Kim, J.O. Kim, A. Urbas, S.J. Lee, Z. Ku, J. Zhou, A Low-Loss Metasurface Antireflection Coating on Dispersive Surface Plasmon Structure, Sci. Rep. 6 (2016) 36190.

[49] H. Lee, C.L. Soles, H.W. Ro, R.L. Jones, E.K. Lin, W. Wu, D.R. Hines, Nanoimprint Pattern Transfer Quality from Specular X-Ray Reflectivity, Appl. Phys. Lett. 87 (2005) 263111.

[50] G. Zhen, P. Zhou, X. Luo, J. Xie, L. Deng, Modes Coupling Analysis of Surface Plasmon Polaritons Based Resonance Manipulation in Infrared Metamaterial Absorber, Sci. Rep. 7 (2017) 46093.

[51] G. Xu, Z. Shi, Y. Zhao, J. Deng, M. Dong, C. Liu, V Murugadoss, X Mai, Z. Guo, Structural Characterization of Lignin and its Carbohydrate Complexes Isolated from Bamboo (Dendrocalamus sinicus), International J. Biological Macromolecules, 126 (2019) 376-384.

[52] D. Jiang, Y. Wang, B. Li, C. Sun, Z. Wu, H. Yan, L. Xing, S. Qi, Y. Li, H. Liu, W. Wei, X. Wang, T. Ding and Z. Guo, Flexible Sandwich Structural Strain Sensor based on Silver Nanowires Decorated Self-healing Substrate, Macromol. Mater. Eng., 2019, in press, DOI: 10.1002/mame.201900074.

[53] Z. Shi, C. Jia, D. Wang, J. Deng, G. Xu, C. Wu, M. Dong, and Z. Guo, Synthesis and Characterization of Porous Tree Gum Grafted Copolymer Derived from Prunus Cerasifera Gum Polysaccharide, International J. Biological Macromolecules, 133 (2019) 964-970.

[54] Z. Hu, D. Zhang, F. Lu, W. Yuan, X. Xu, Q. Zhang, H. Liu, Q. Shao, Z. Guo, Y. Huang, Multistimuli-responsive intrinsic self-healing epoxy resin constructed by host-guest interactions. Macromolecules 51 (2018) 5294-5303.

[55] Z. Hu, Y. Liu, X. Xu, W. Yuan, L. Yang, Q. Shao, Z. Guo, T. Ding, Y. Huang. Efficient Intrinsic Self-healing Epoxy Acrylate Formed from Host-guest Chemistry. Polymer, 164 (2019) 79-85. 


\section{Highlights}

- The first polymer-based mid-wavelength infrared linear polarizer was built with sulfuric polymer film.

- Current mid-wavelength infrared polarizer optical elements are based on expensive and fragile inorganic materials.

- Transmission and extinction ratio are comparable to commercial products.

- Both computational simulation and analytical model confirmed the enhanced transmission efficiency and extinction ratio.

- Advantages of the polymeric material allow low-cost and scalable manufacturing 